Supporting information

\title{
Exploiting Deep Eutectic Solvents and Ionic Liquids for the valorization of Chestnut Shell Waste
}

Elena Husanu, ${ }^{\dagger,+}$ Angelica Mero, ${ }^{\dagger,+}$ Jose Gonzalez Rivera, ${ }^{\dagger}, \ddagger$ Andrea Mezzetta, ${ }^{\dagger}$

Julian Cabrera Ruiz,§ Felicia D’Andrea, † Christian Silvio Pomelli, † Lorenzo Guazzelli t, ${ }^{*}$

† Department of Pharmacy, University of Pisa, Via Bonanno 33, 56126 Pisa, Italy

‡ National Institute of Optics, (INO-CNR)-UOS Pisa, Via G. Moruzzi 1, 56124 Pisa, Italy

$\S$ Department of Chemical Engineering, University of Guanajuato, Noria Alta s/n, 36050, Guanajuato, Gto, Mexico.

+ Elena Husanu and Angelica Mero equally contributed to this work.

* corresponding author: lorenzo.guazzelli@unipi.it 
Schematic representations of hydrolysable and condensed tannins and simple phenolic acids page S3

Schematic representations of cellulose and hemicellulose page $S 4$

Schematic representations of Lignin and phenyl propanoid monomers page S5

${ }^{1} \mathrm{H}-\mathrm{NMR}$ spectra of prepared DESs and bio-IL pages S6-S14

1H-NMR spectra of prepared and recovered $\mathrm{ChCl}: \mathrm{O} a x 2 \mathrm{H} 2 \mathrm{O}$ page S15

HPLC chromatogram and retention times of polyphenol standards page S16

1H-NMR of spectrum of prepared and recovered bio-IL page S17

Pyrolysis steps using the second derivative method page $\mathrm{S} 18$

HPLC chromatograms of extracts using different DESs pages S19-S20

Picture of different Polyphenol extracts in methanol page $S 21$

TG and DTG simulation results of three-parallel-DAEM model pages S22-S23

Yields of extracts obtained using different DESs page S24

Interval range experimental data Identified by DDTG method to fit the three parallel-DAEM model page S24

Optimized kinetics parameters of first order three-DAEM-reaction model page $\mathbf{S} 25$ 


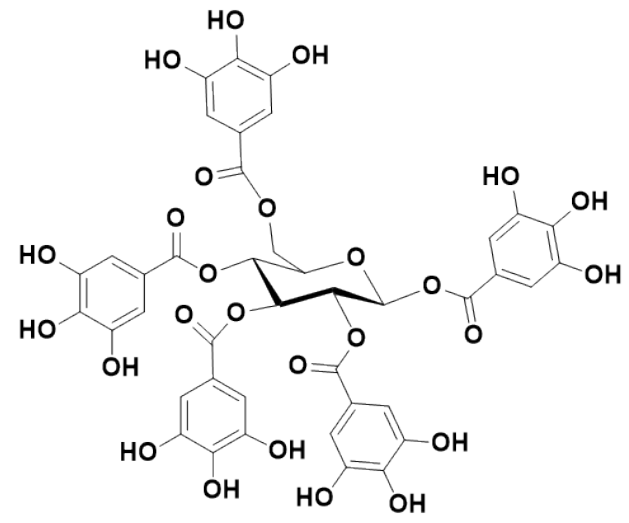

Hydrolizable tannins
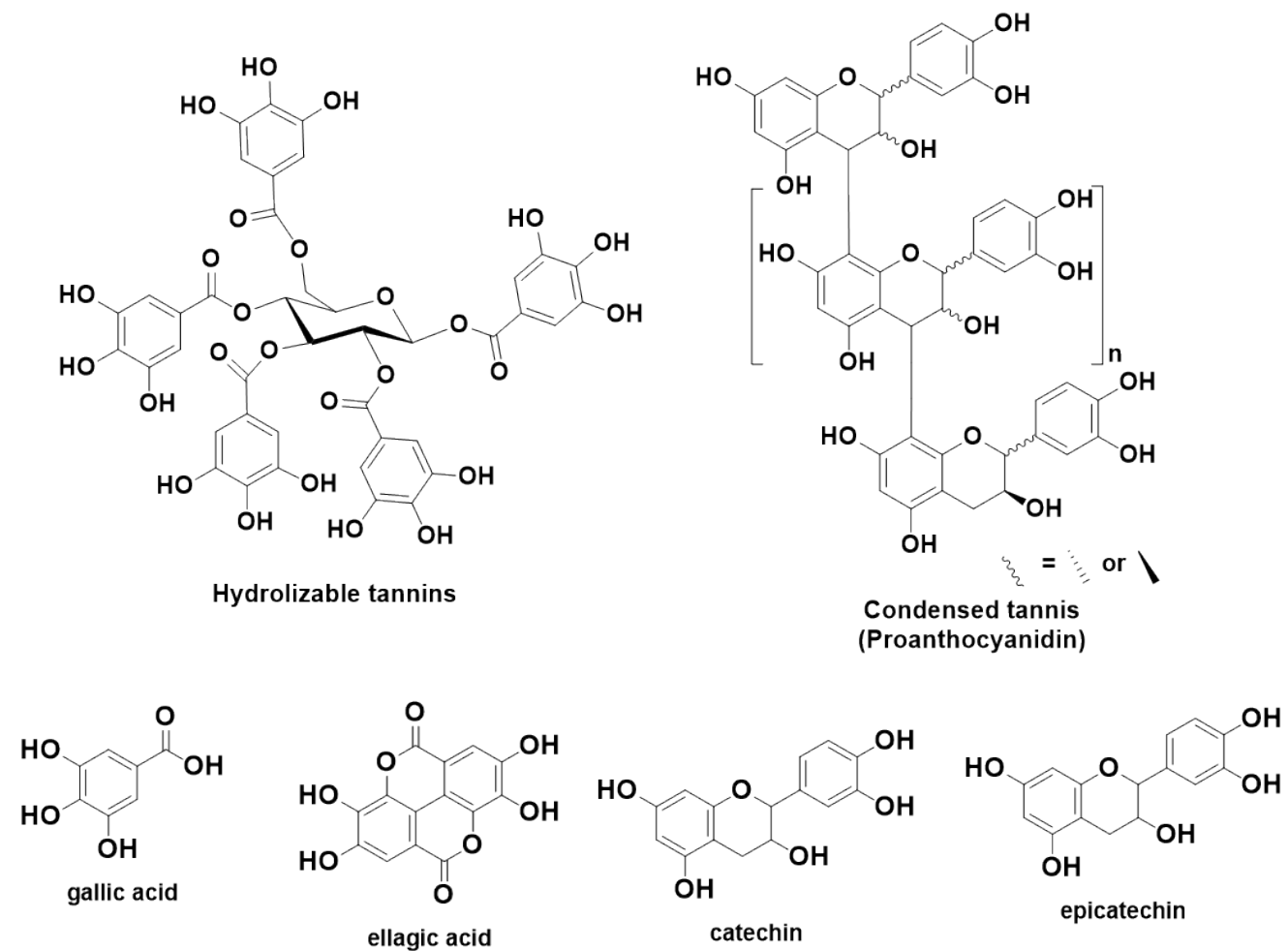

Figure S1. Representative structures of hydrolysable and condensed tannins and simple phenolic acids 


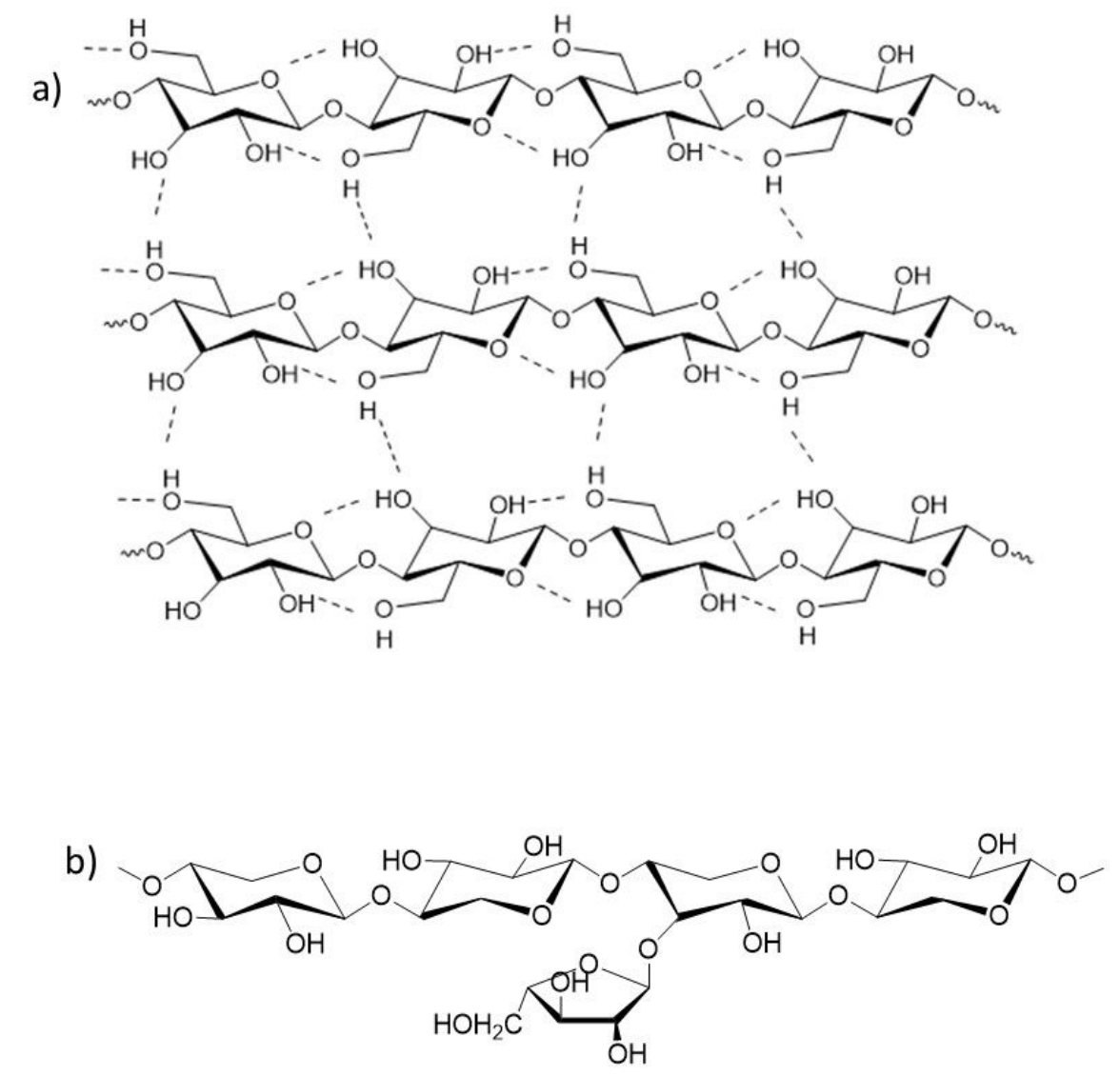

Figure S2. a) Cellulose structure with hydrogen bonds and b) an example of hemicellulose structure 


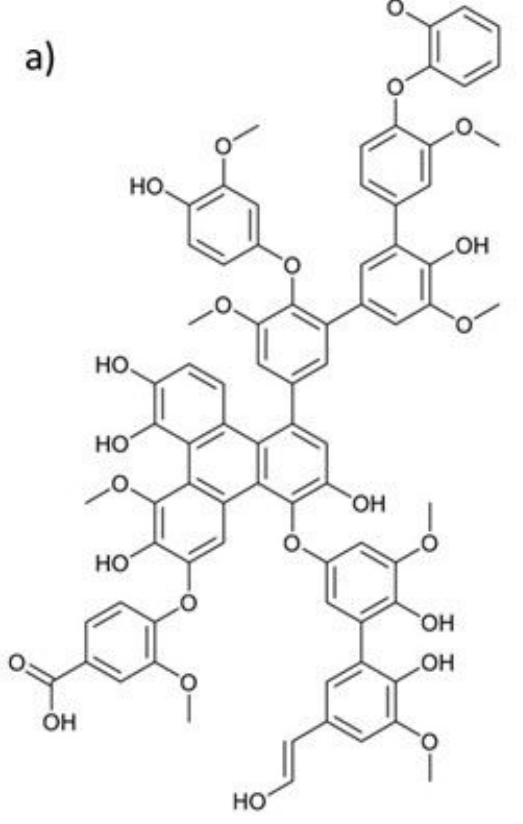

b)<smiles>COc1cc(/C=C/CO)cc(OC)c1O</smiles>

\section{Sinapyl alchol}

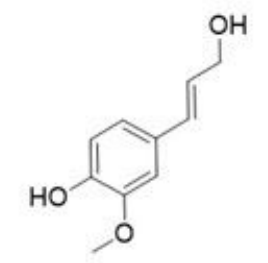

Guaiacyl alchol

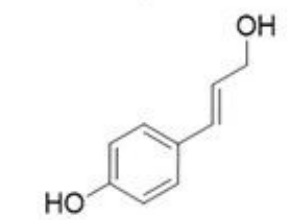

p-Coumaryl alchol

Figure S3. a) Typical lignin structure and b) phenyl propanoid monomers 


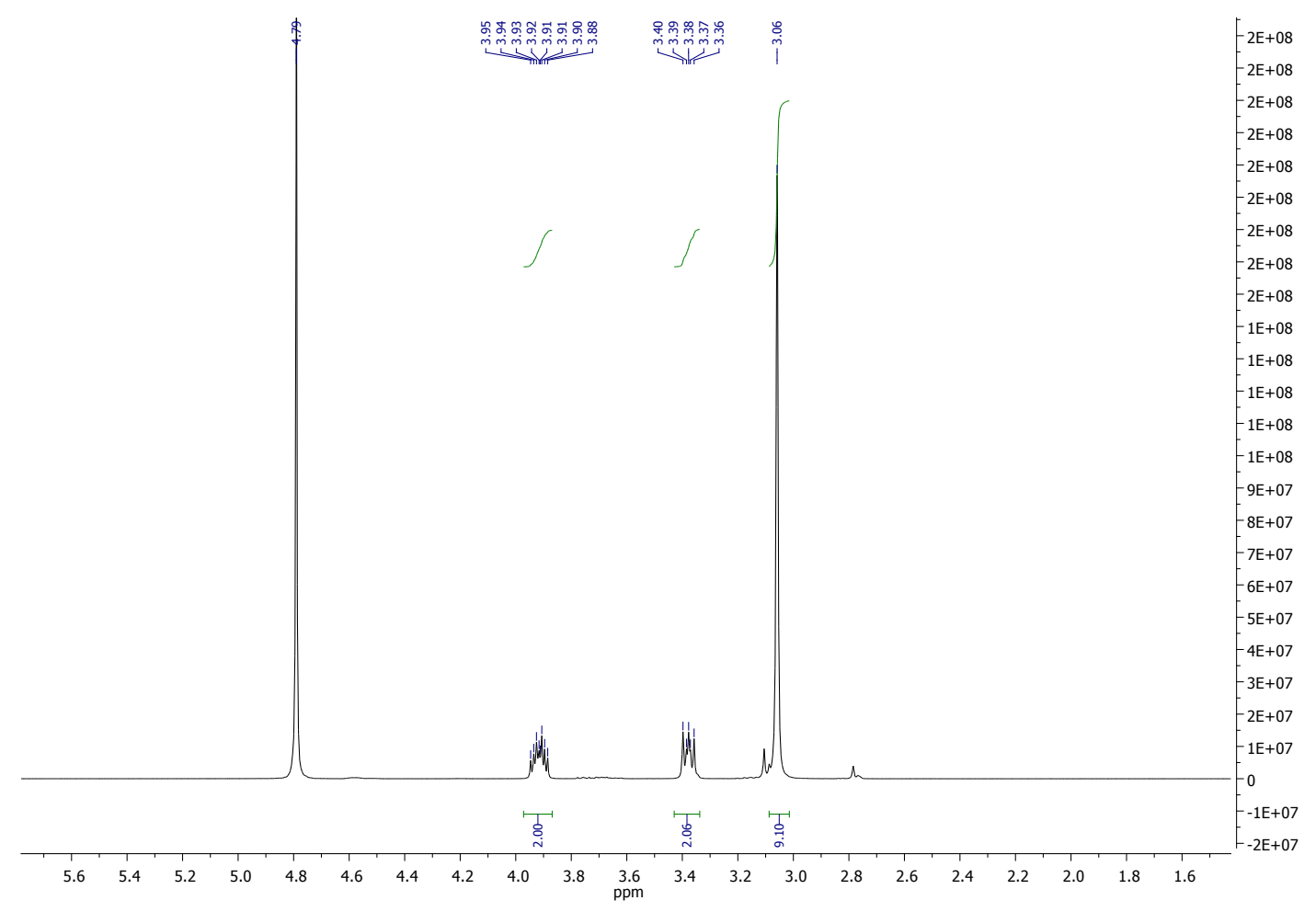

Figure S4. ${ }^{1} \mathrm{H}-\mathrm{NMR}$ spectrum $\left(\mathrm{D}_{2} \mathrm{O}, 400 \mathrm{MHz}\right)$ of $\mathrm{ChCl}: \mathrm{Oax} 2 \mathrm{H}_{2} \mathrm{O}$ 


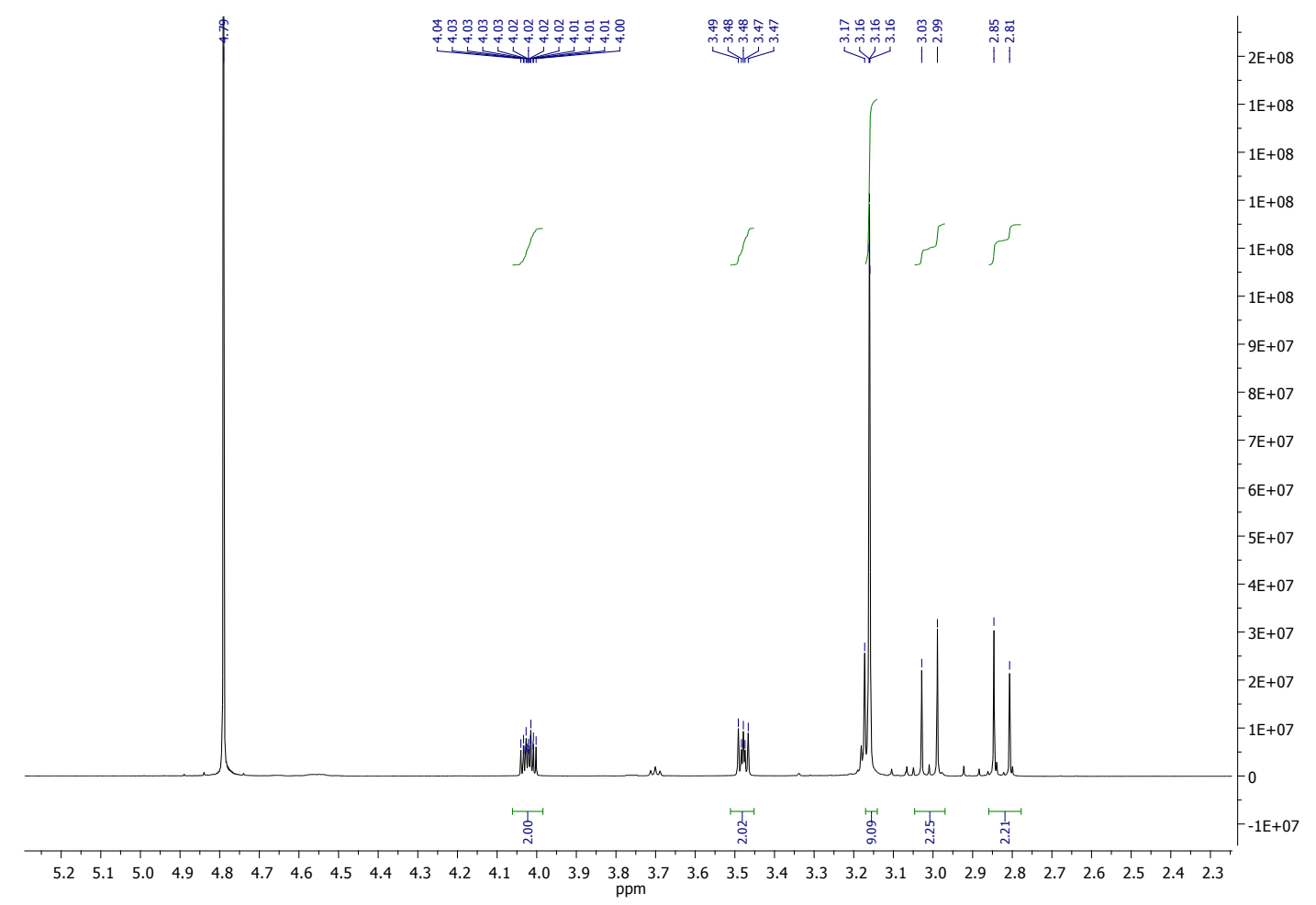

Figure S5. ${ }^{1} \mathrm{H}-\mathrm{NMR}$ spectrum $\left(\mathrm{D}_{2} \mathrm{O}, 400 \mathrm{MHz}\right)$ of $\mathrm{ChCl}: \mathrm{CaxH}_{2} \mathrm{O}(1: 1)$ 


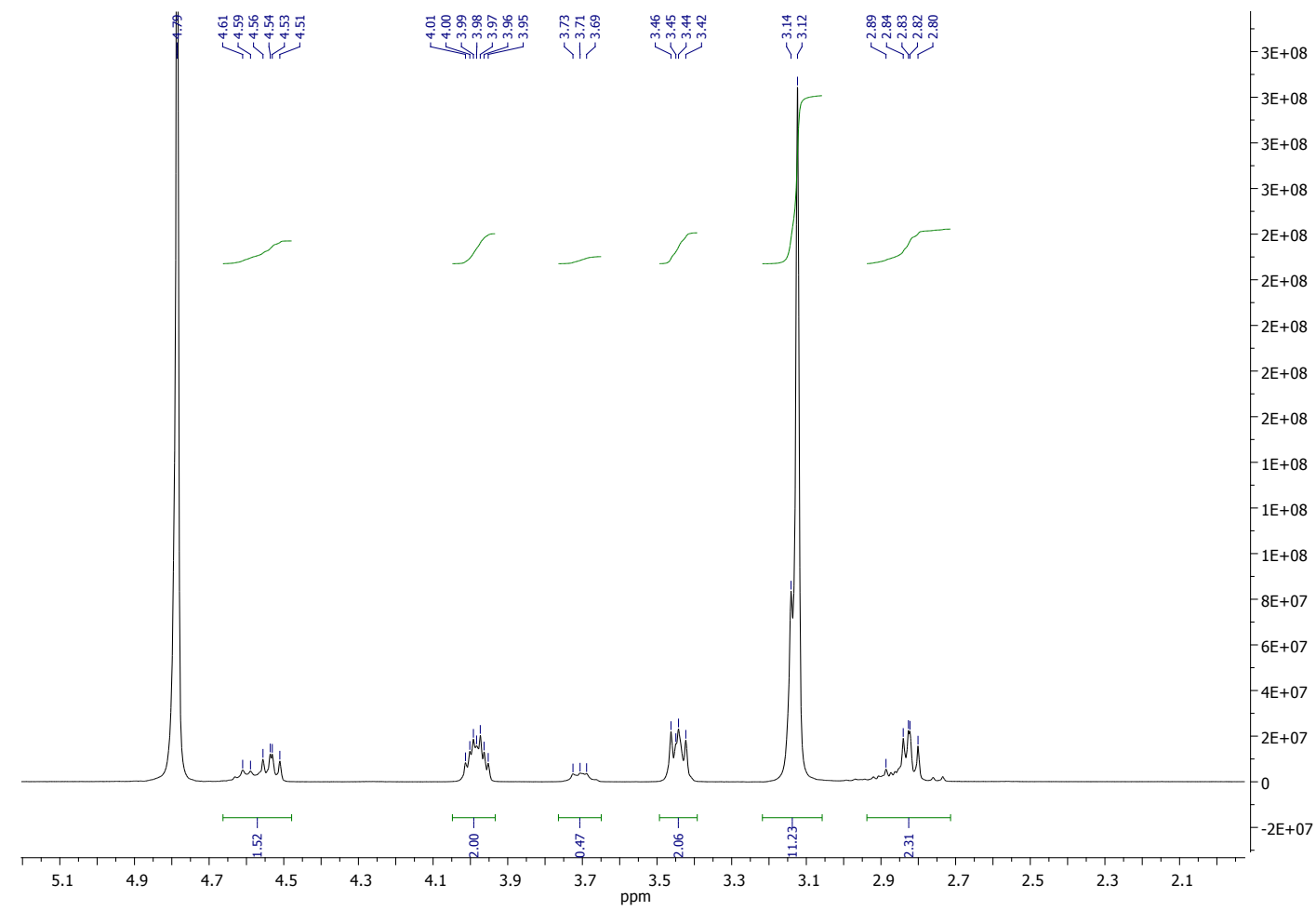

Figure S6. ${ }^{1} \mathrm{H}-\mathrm{NMR}$ spectrum $\left(\mathrm{D}_{2} \mathrm{O}, 400 \mathrm{MHz}\right)$ of $\mathrm{ChCl}: \mathrm{Ma}(1: 1)$ 


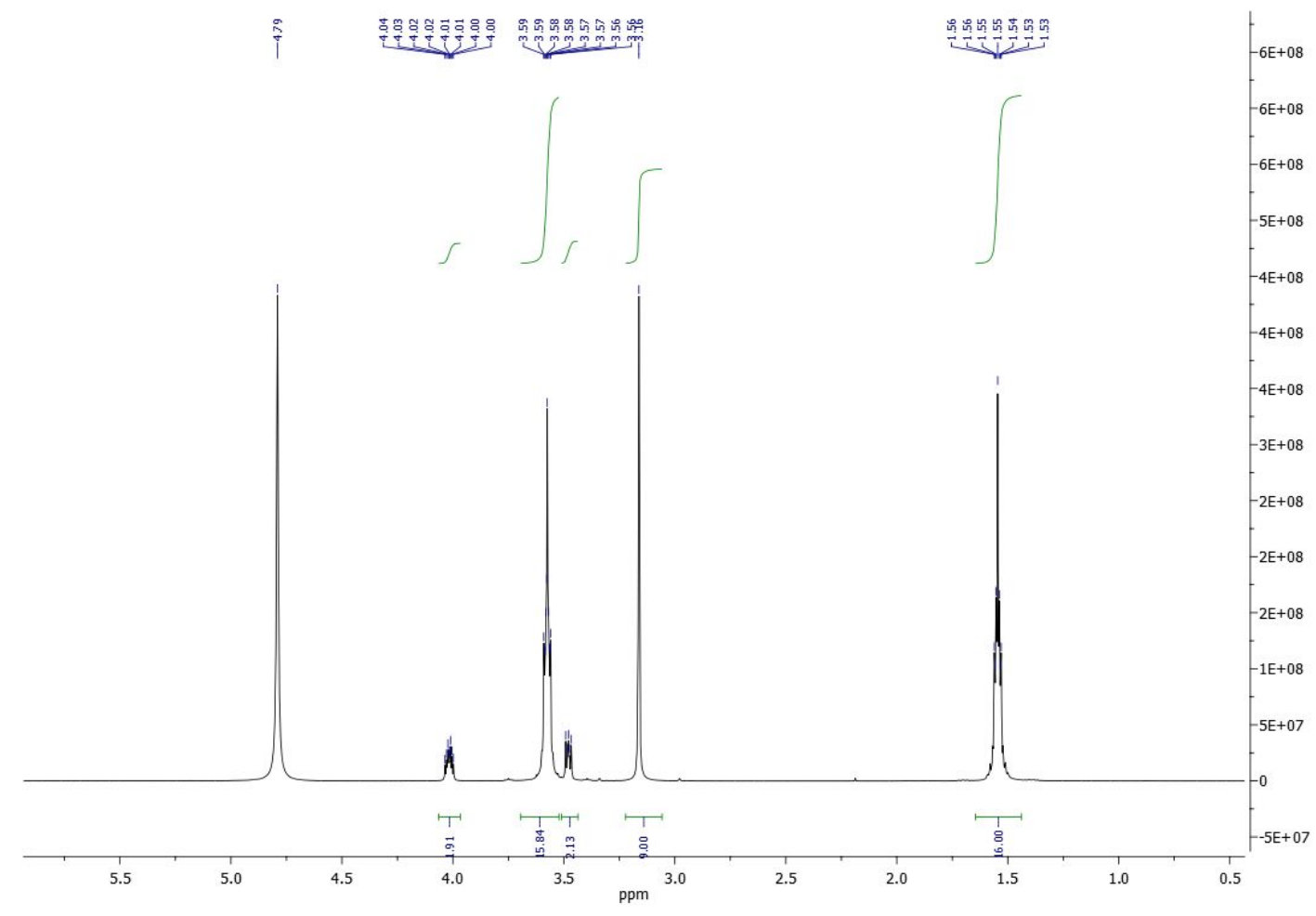

Figure S7. ${ }^{1} \mathrm{H}-\mathrm{NMR}$ spectrum $\left(\mathrm{D}_{2} \mathrm{O}, 400 \mathrm{MHz}\right)$ of $\mathrm{ChCl}$ : But $(1: 4)$ 


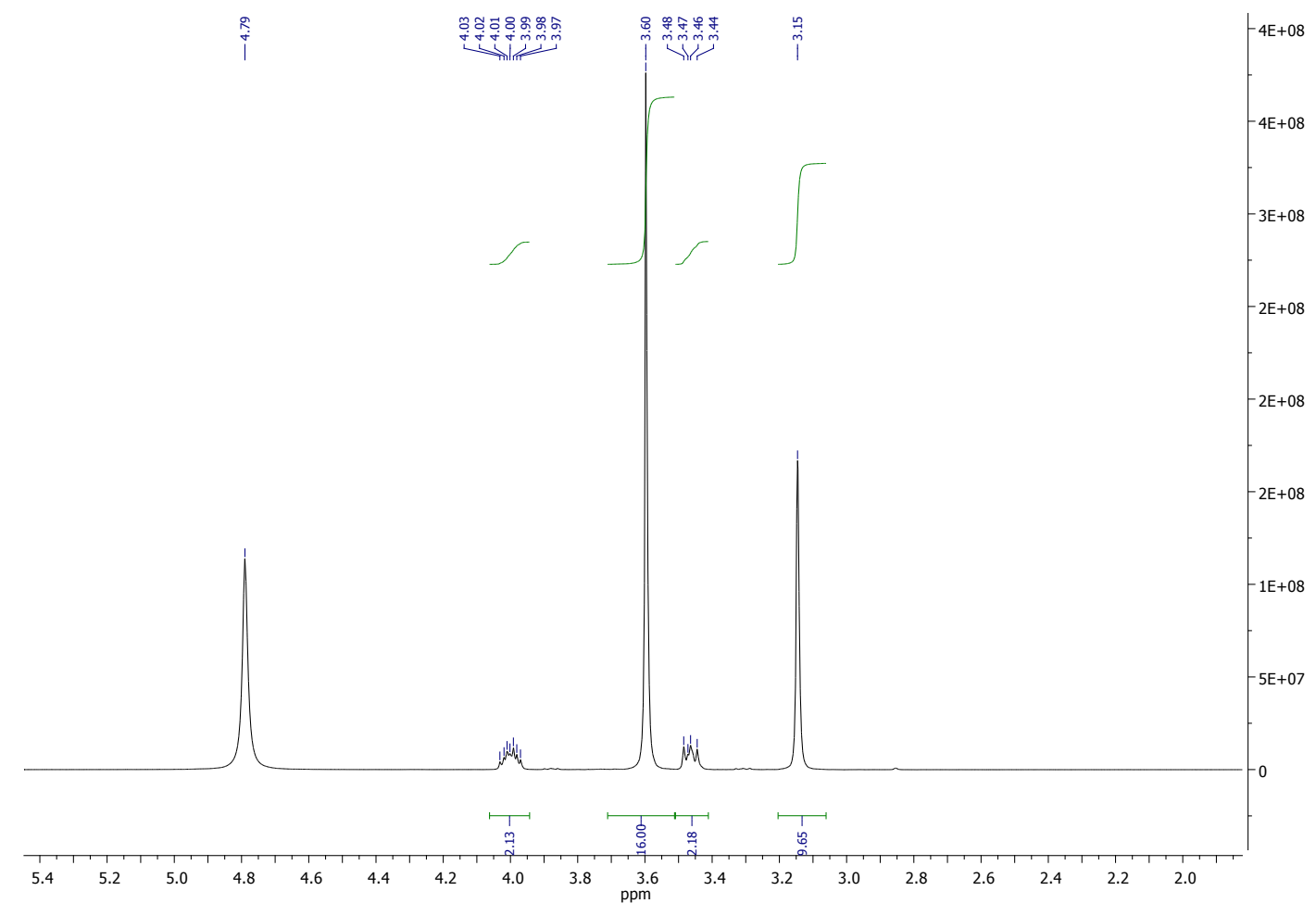

Figure S8. ${ }^{1} \mathrm{H}-\mathrm{NMR}$ spectrum $\left(\mathrm{D}_{2} \mathrm{O}, 400 \mathrm{MHz}\right)$ of $\mathrm{ChCl}$ :EG (1:4) 


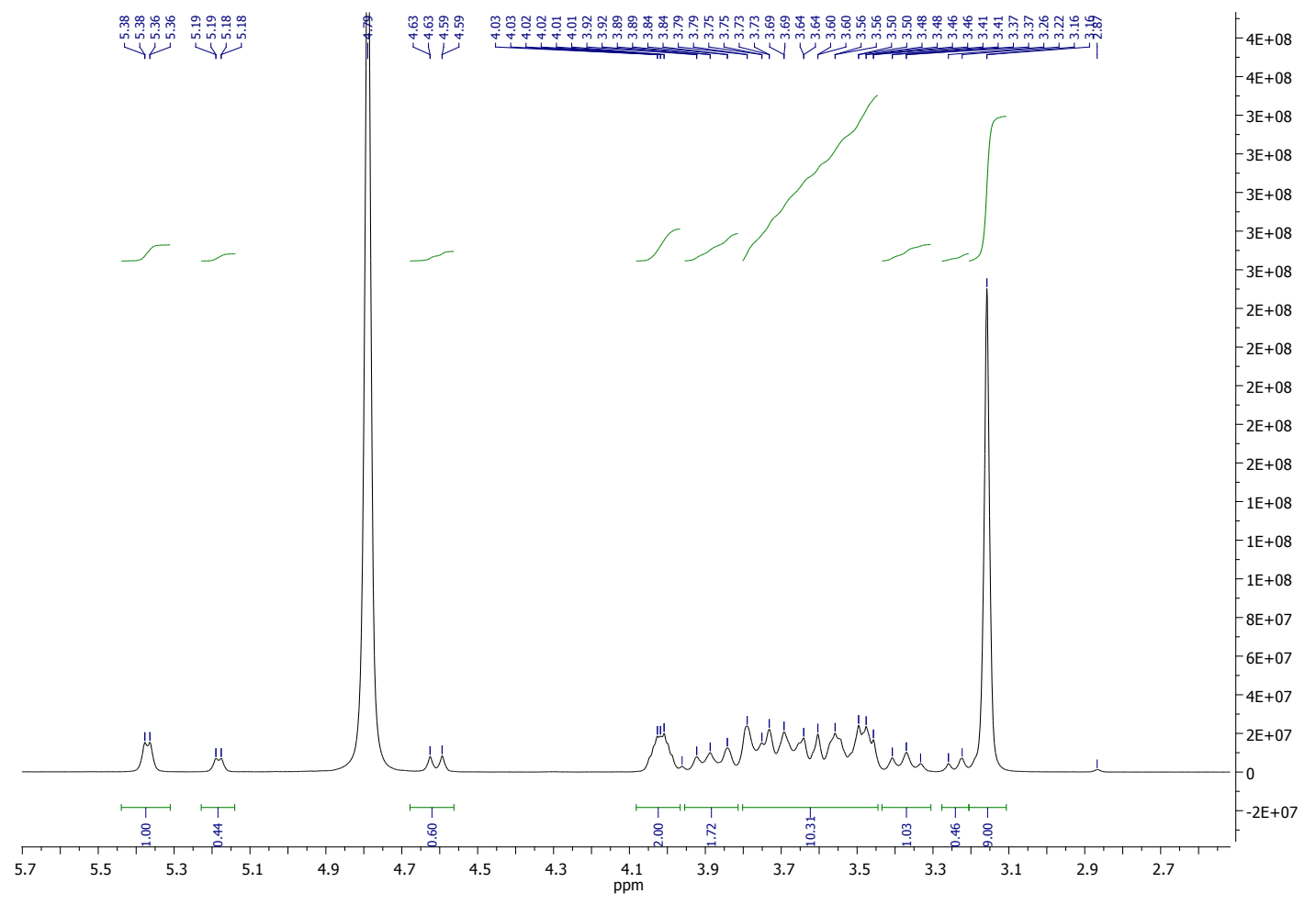

Figure S9. ${ }^{1} \mathrm{H}-\mathrm{NMR}$ spectrum $\left(\mathrm{D}_{2} \mathrm{O}, 400 \mathrm{MHz}\right)$ of $\mathrm{ChCl}$ :Mal (1:1) 


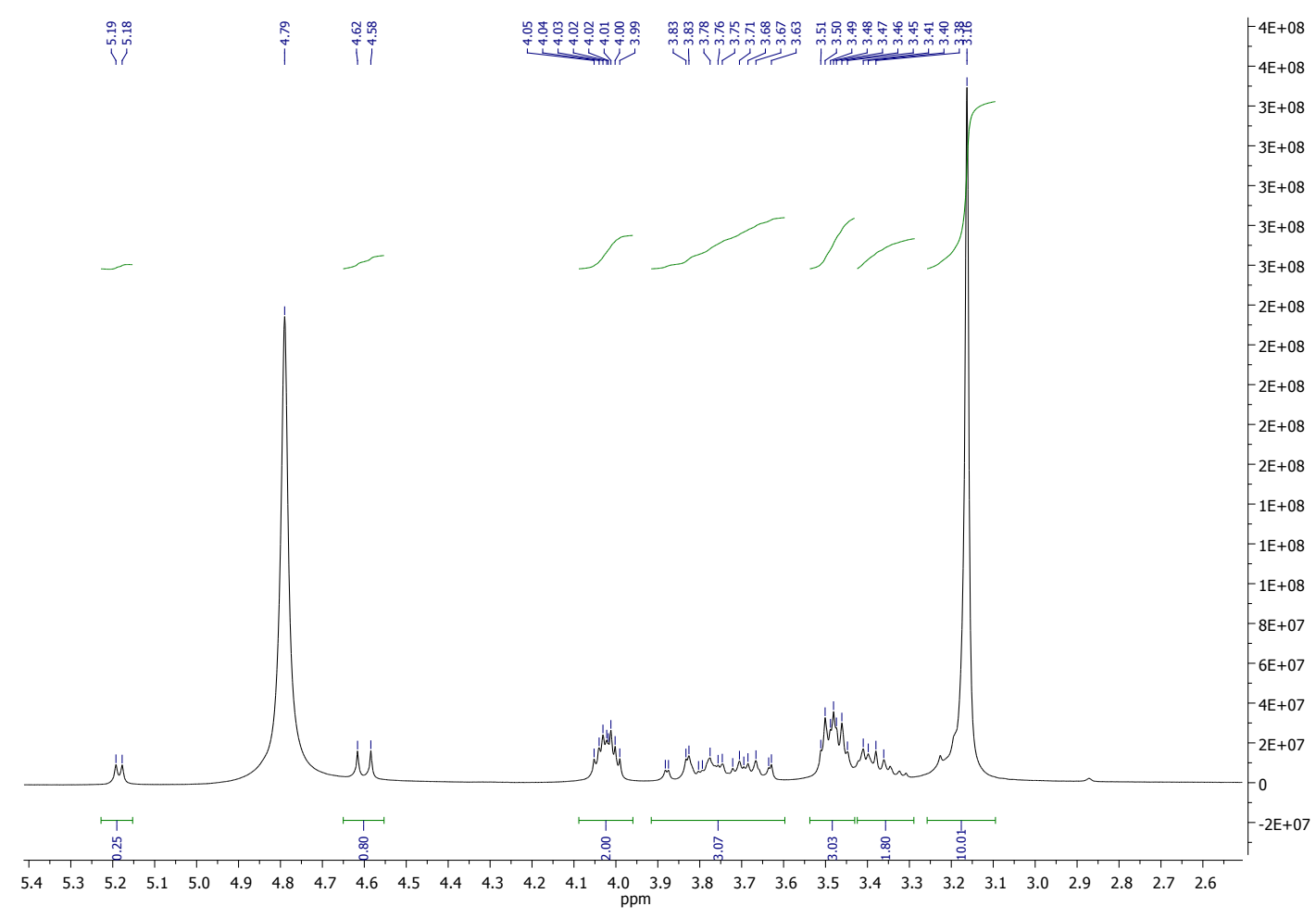

Figure S10. ${ }^{1} \mathrm{H}-\mathrm{NMR}$ spectrum $\left(\mathrm{D}_{2} \mathrm{O}, 400 \mathrm{MHz}\right)$ of $\mathrm{ChCl}: \mathrm{Glu}(1: 1)$ 


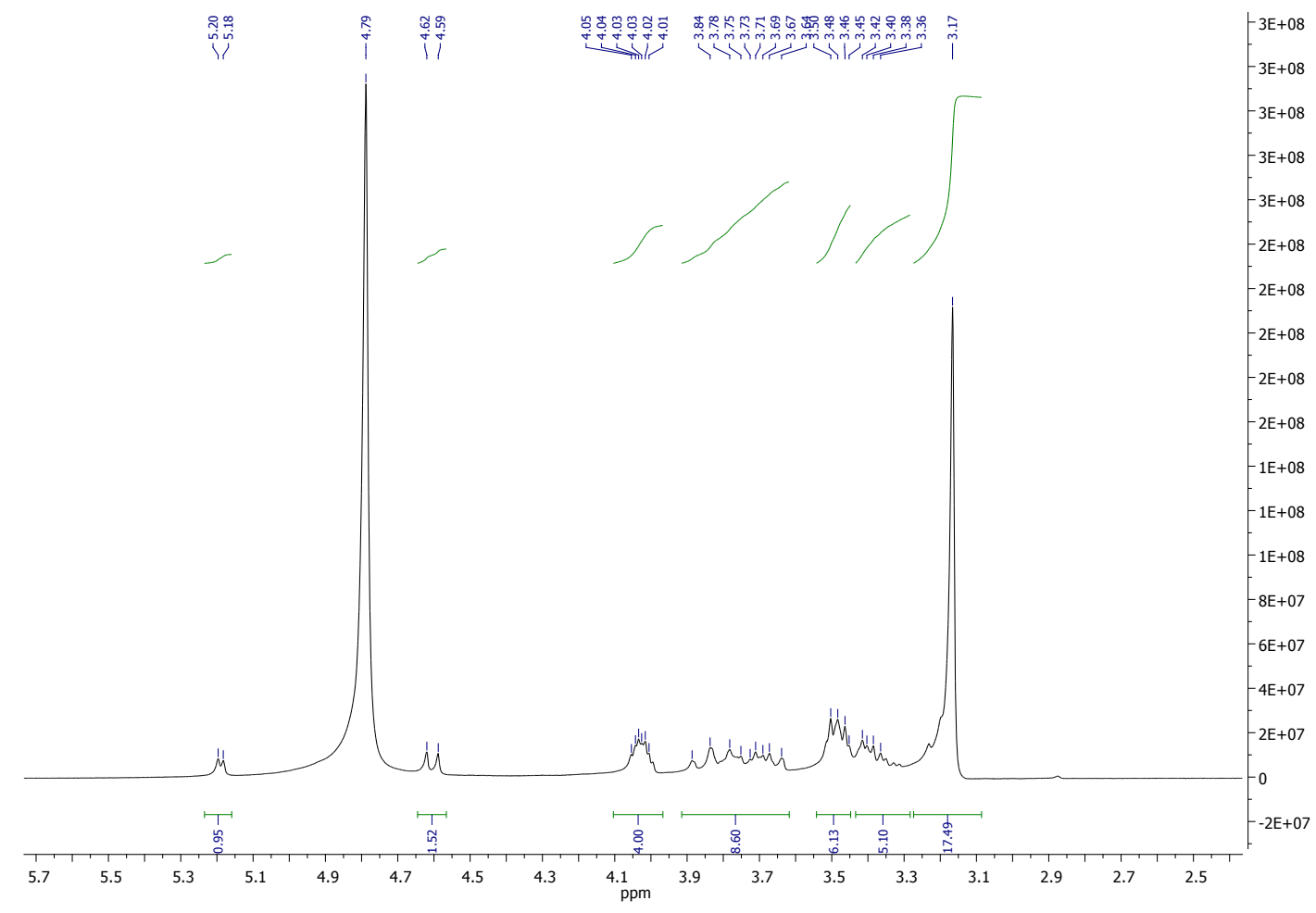

Figure S11. ${ }^{1} \mathrm{H}-\mathrm{NMR}$ spectrum $\left(\mathrm{D}_{2} \mathrm{O}, 400 \mathrm{MHz}\right)$ of $\mathrm{ChCl}: \mathrm{Glu}(1: 2)$ 


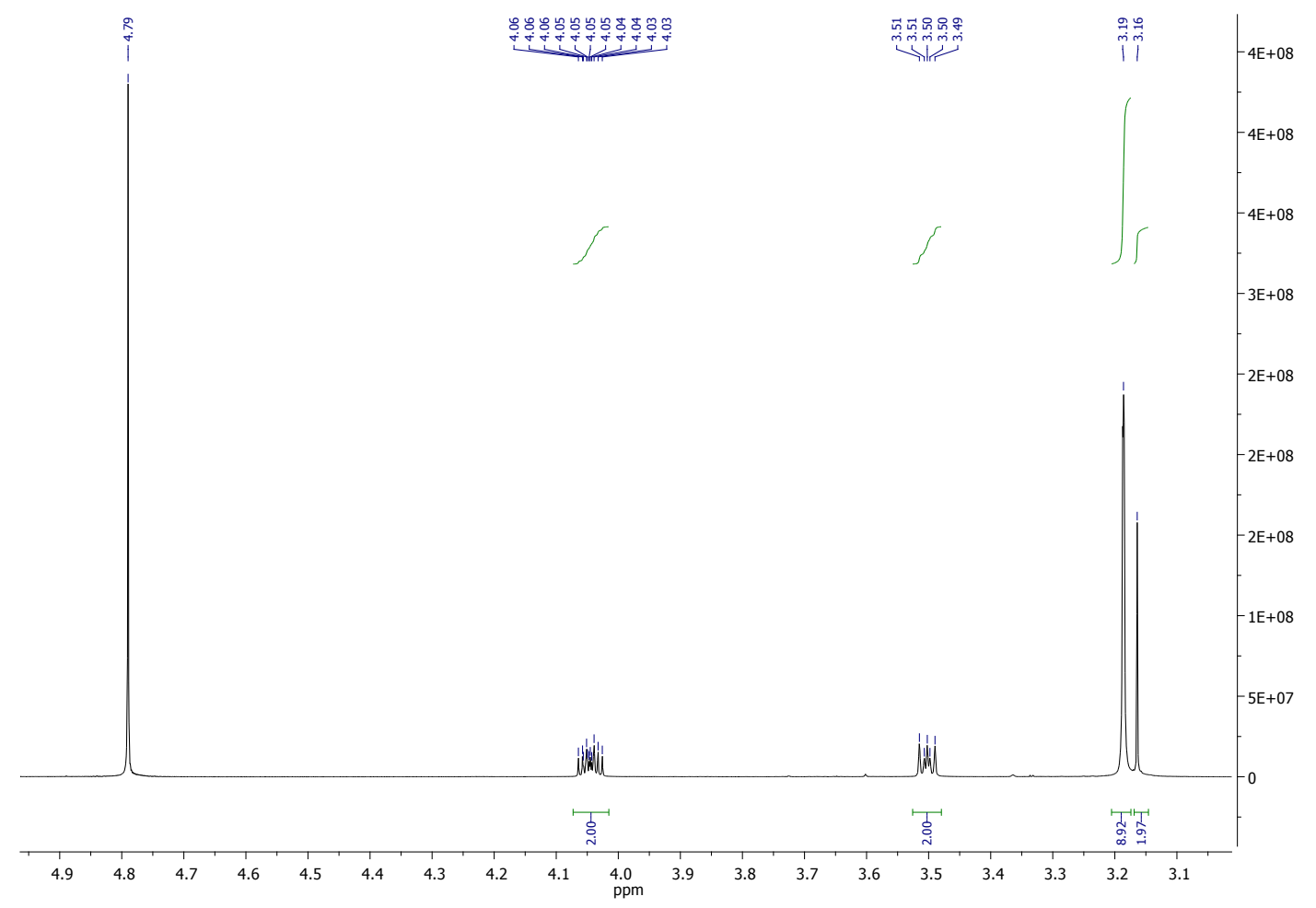

Figure S12. ${ }^{1} \mathrm{H}-\mathrm{NMR}$ spectrum $\left(\mathrm{D}_{2} \mathrm{O}, 400 \mathrm{MHz}\right)$ of ChGly 


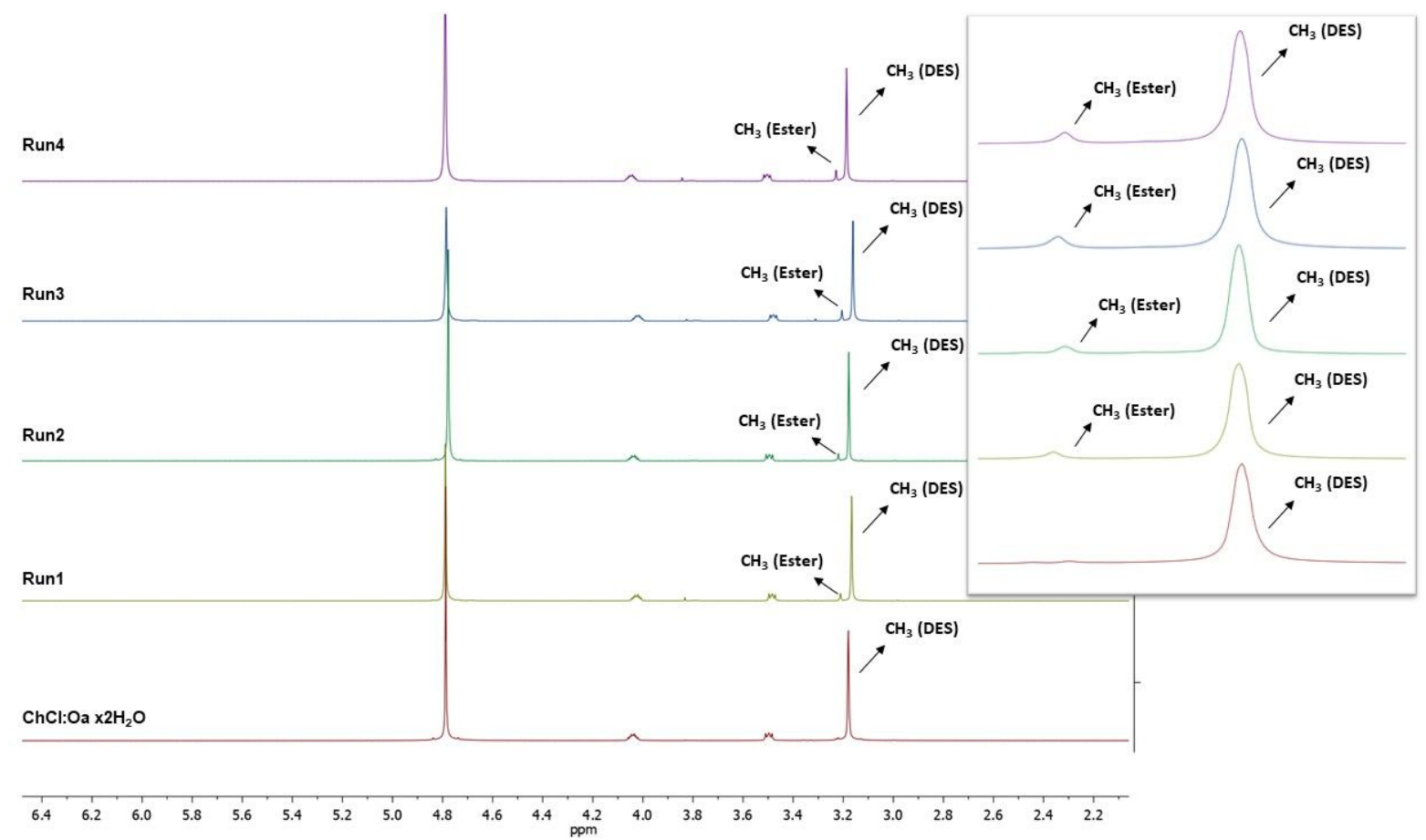

Figure S13. ${ }^{1} \mathrm{H}-\mathrm{NMR}$ spectrum $\left(\mathrm{D}_{2} \mathrm{O}, 400 \mathrm{MHz}\right)$ of $\mathrm{ChCl}: \mathrm{Oax} 2 \mathrm{H}_{2} \mathrm{O}$ compared with the DES recovered after each extraction cycle (in the enlargement, signals of the methyl groups of the starting DES and of the forming ester side product are shown) 


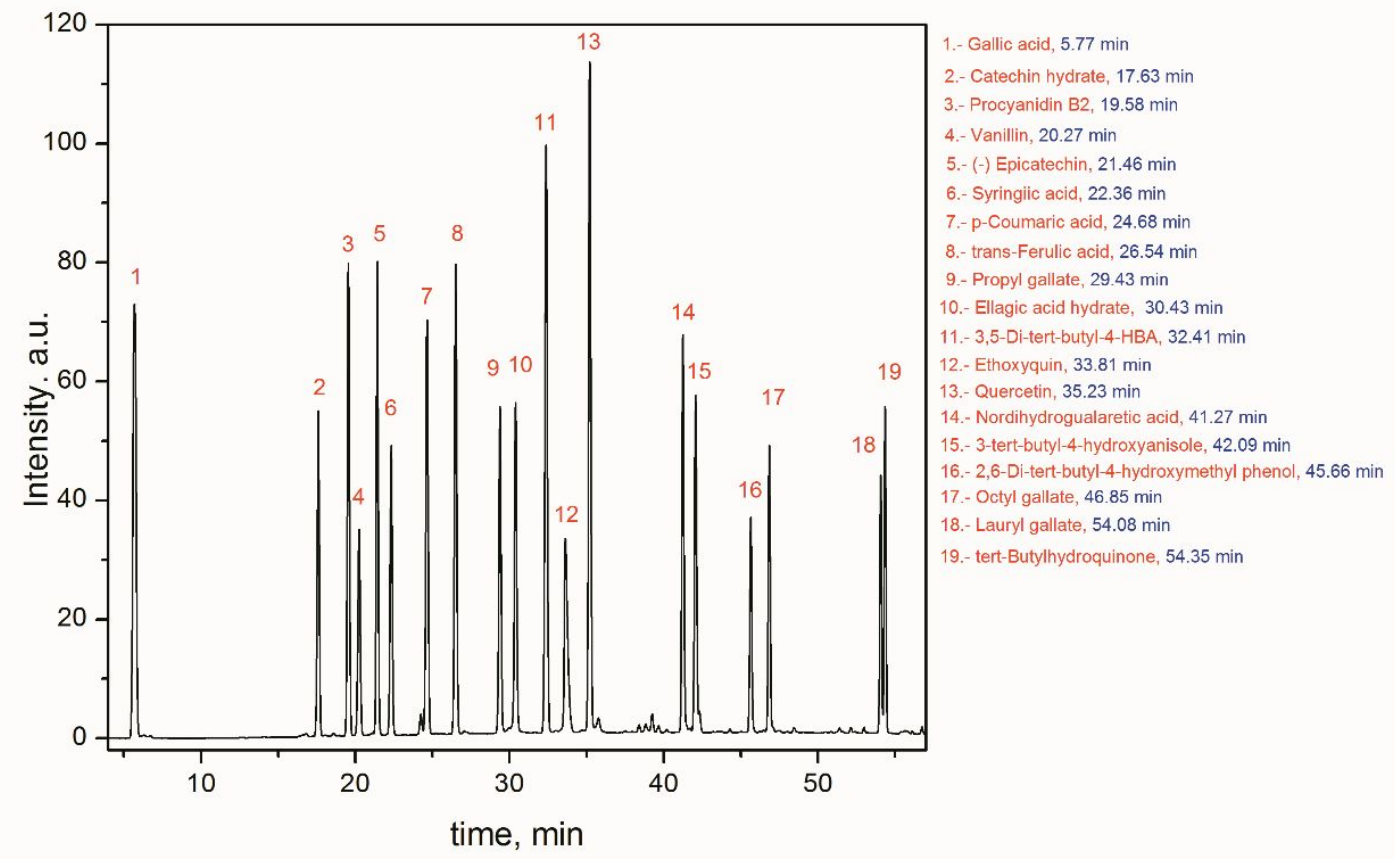

Figure S14. HPLC chromatogram and retention times of polyphenols standards used in this work. 


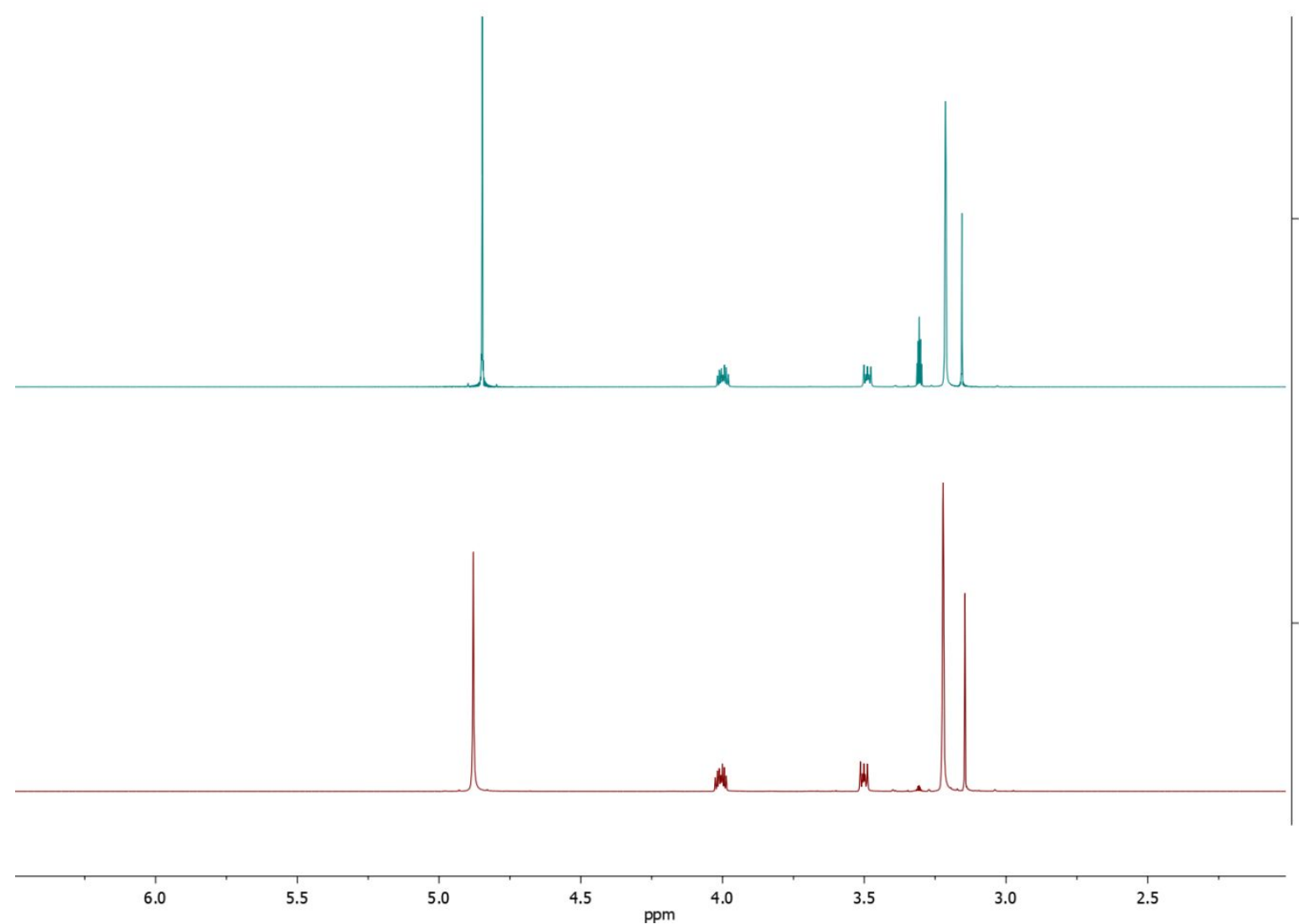

Figure S15. ${ }^{1} \mathrm{H}-\mathrm{NMR}$ spectrum (MeOD, $400 \mathrm{MHz}$ ) of ChGly (bottom, red line) compared with the recovered IL (top, green line) 


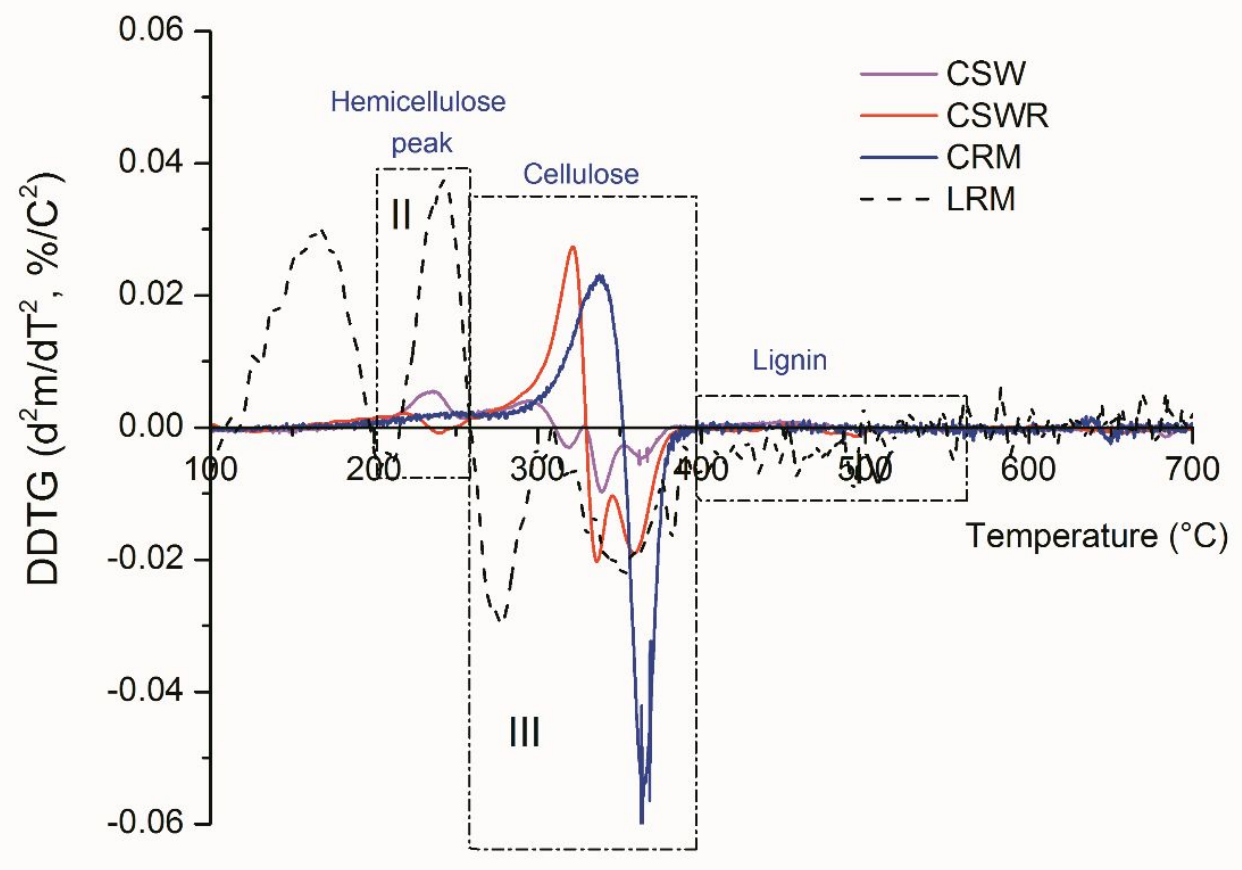

Figure S16. Characteristics of temperature range for the different steps of biomass pyrolysis using the second derivative method. 


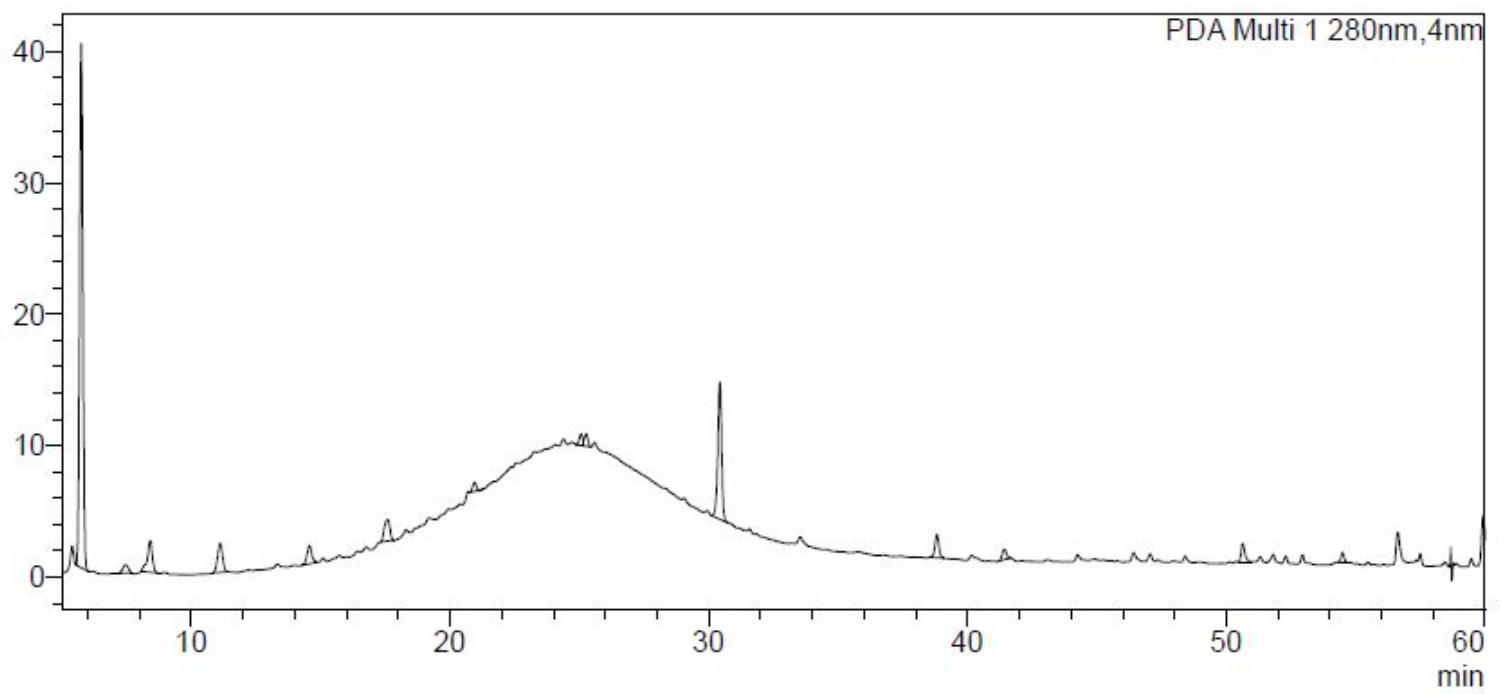

Figure S17. Chromatogram of extract from ChCl:Ma (1:1)

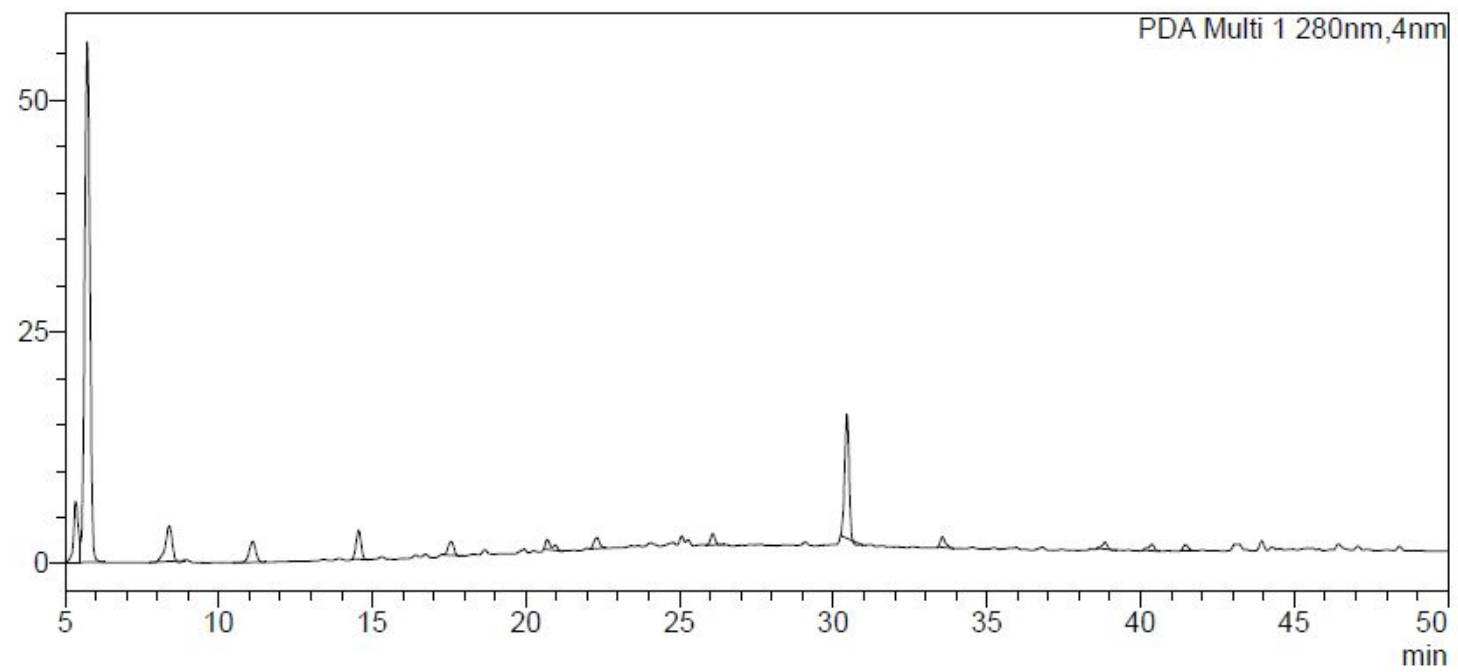

Figure S18. Chromatogram of extract from ChCl: But(1:4) 


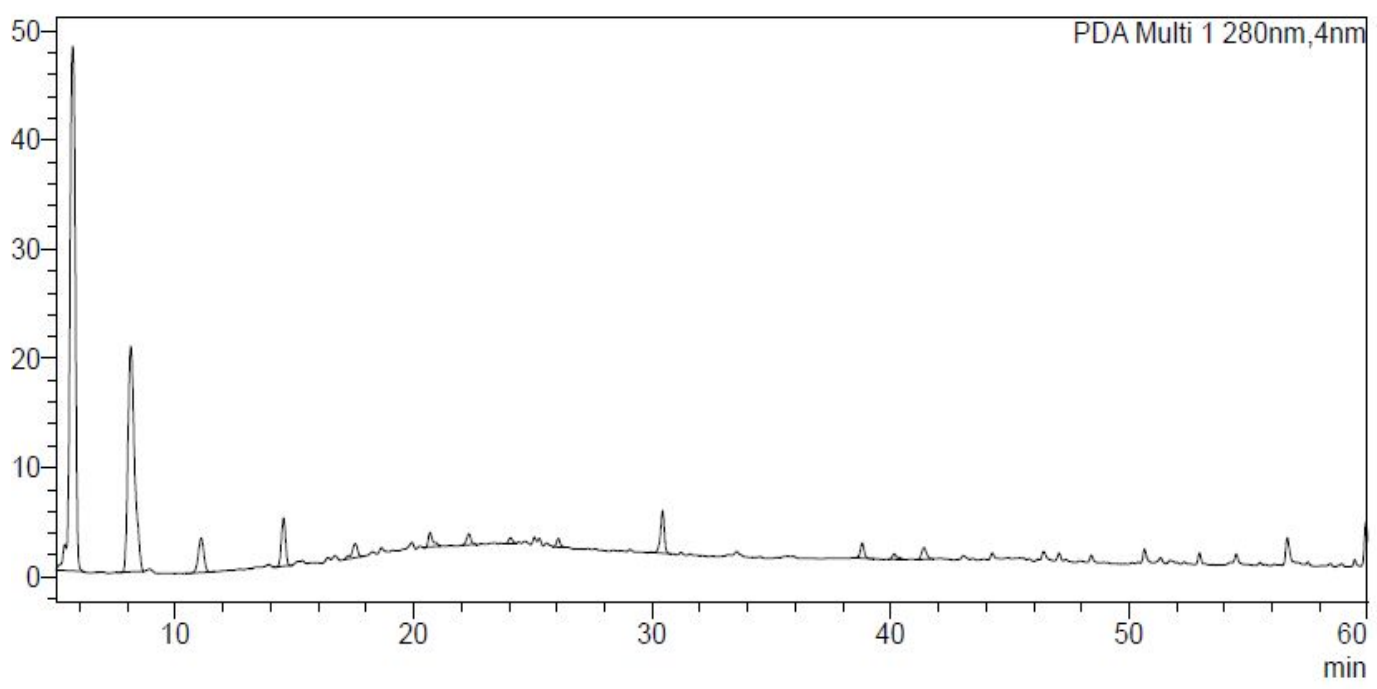

Figure S19. Chromatogram of extract from ChCl:Glu (1:2)

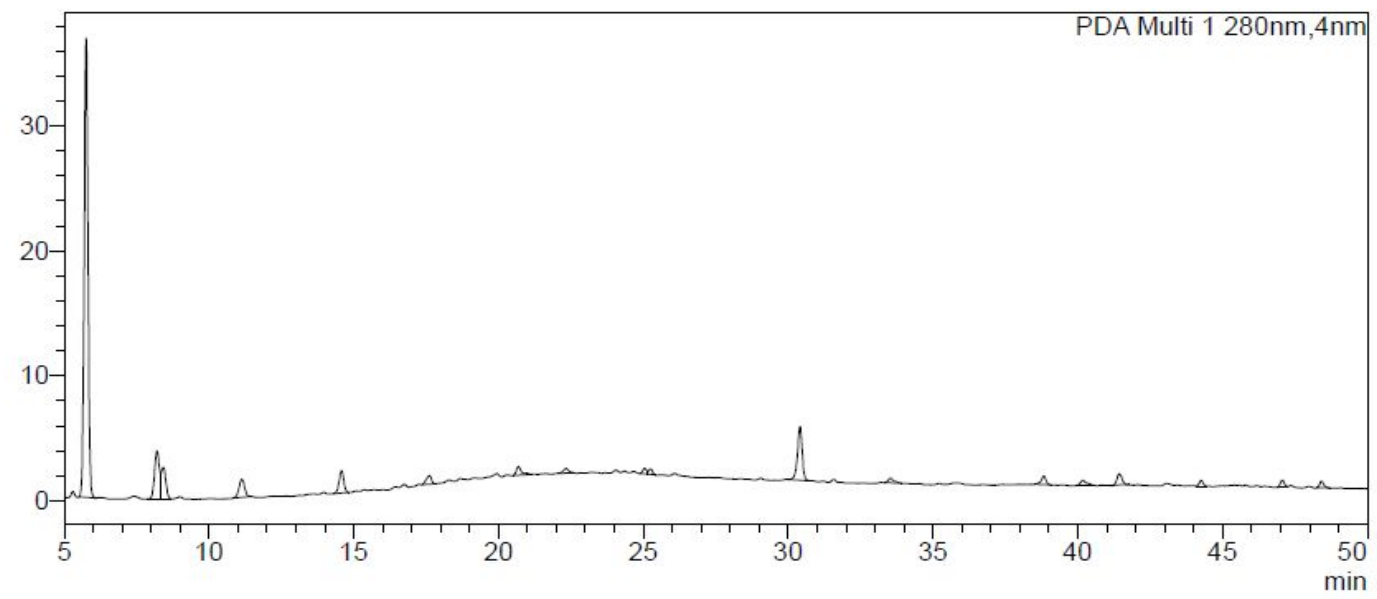

Figure S20. Chromatogram of extract from ChCl:Mal 1:1) 


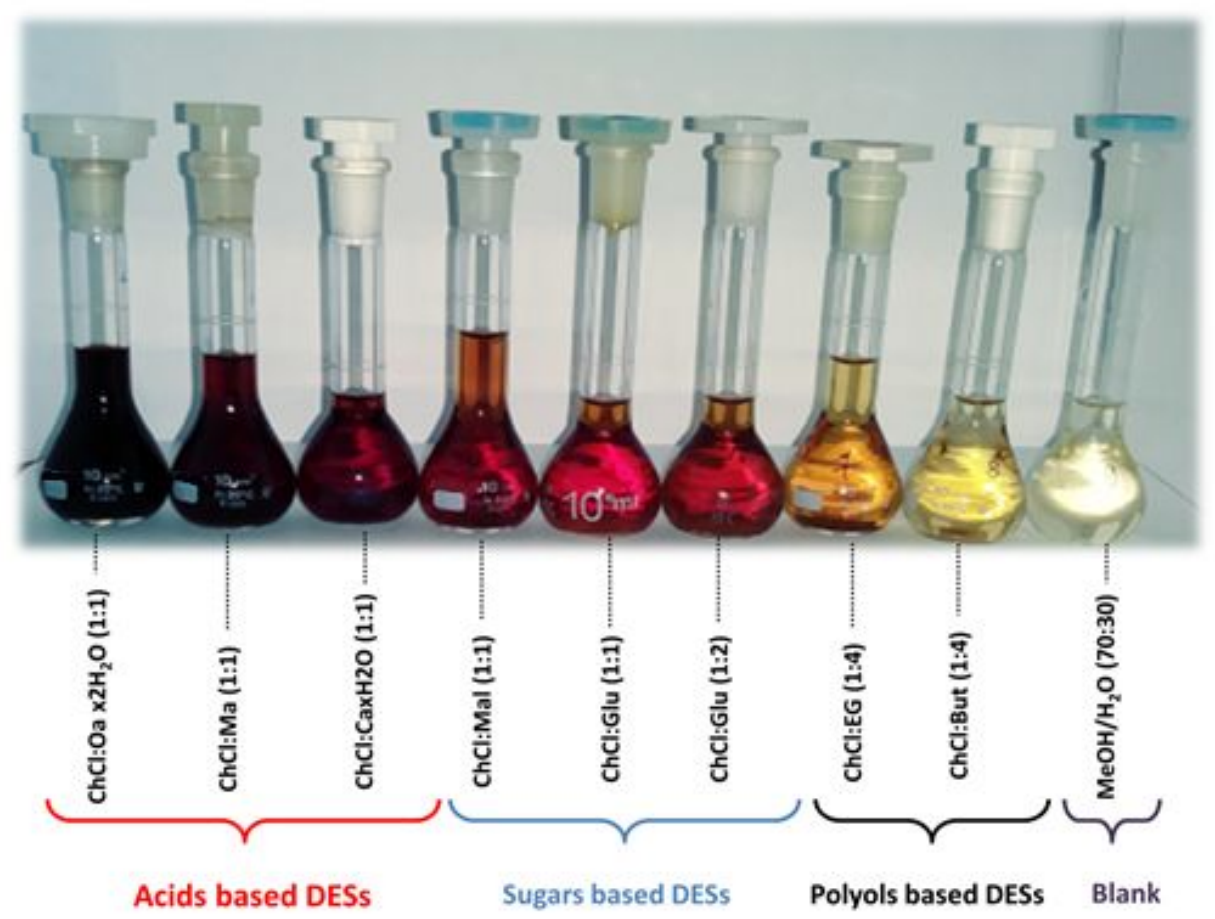

Figure S21. Different color of the polyphenol extracts depending on the extraction DES used (form left to right: acid-based, sugar-based and polyol-based DESs). extraction conditions: $24 \mathrm{~h}$, $65^{\circ} \mathrm{C}, 1: 10 \mathrm{w} / \mathrm{w}$ solid to liquid ratio. 
(a)

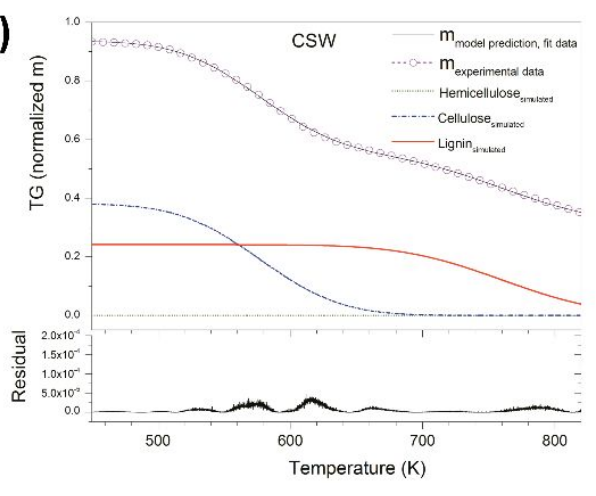

(c)

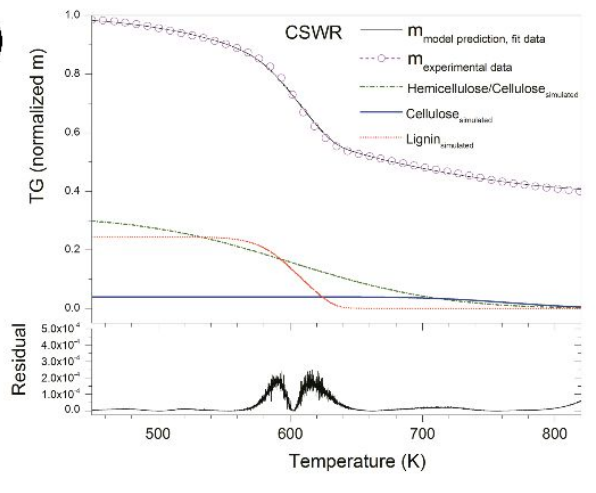

(b)

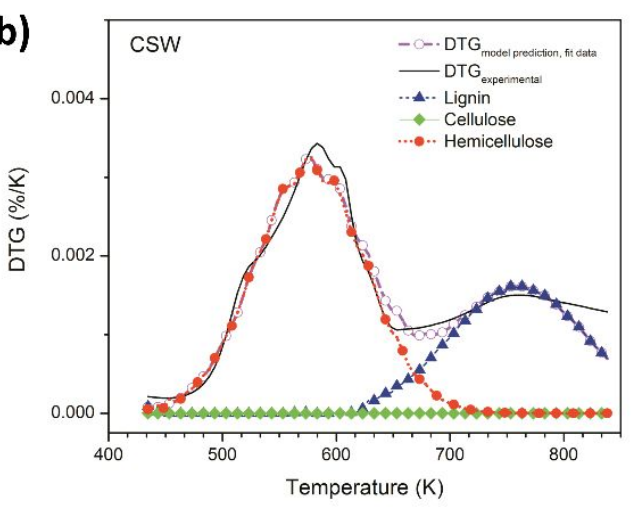

(d)

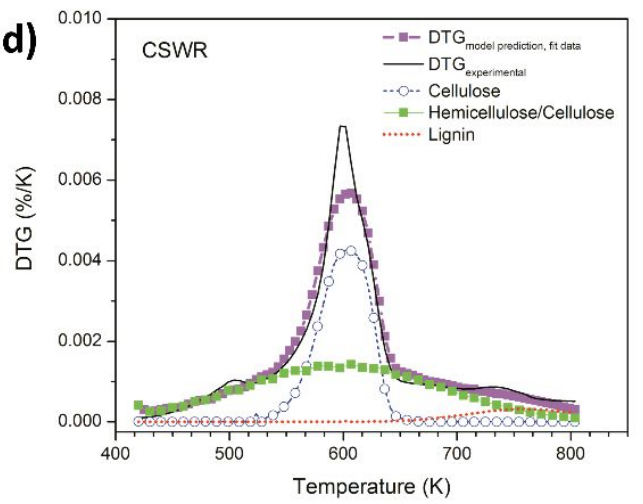

Figure S22. TG $(a, c)$ and DTG (b, d) simulation results of three-parallel-DAEM model for CSW and CSWR, respectively. The residuals between the observed and calculated mass data are also plotted for TG curves (a and c). 
(a)

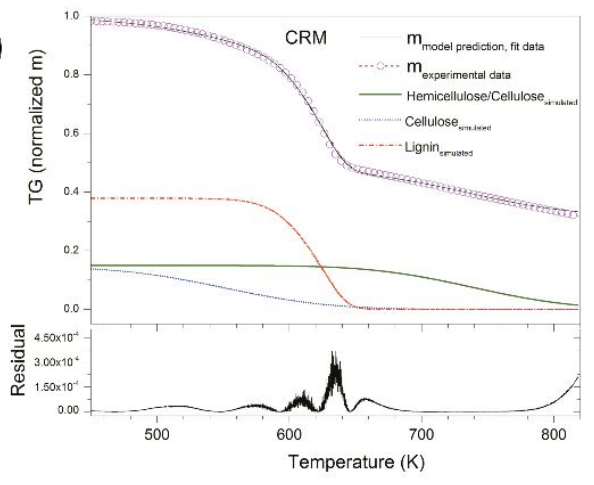

(c)

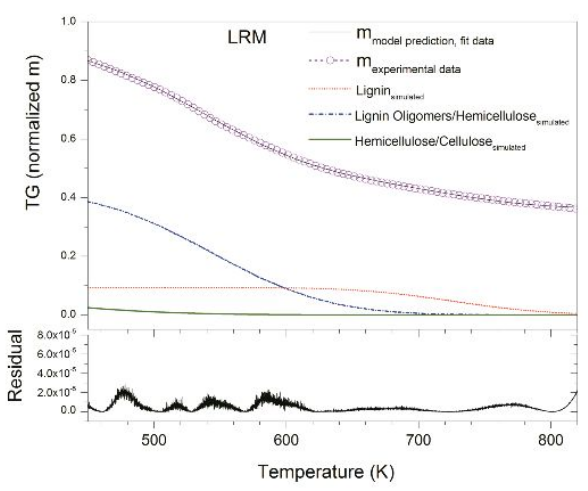

(b)

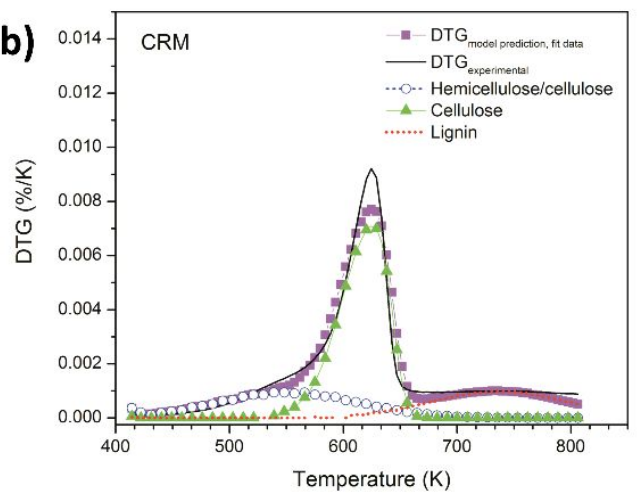

(d)

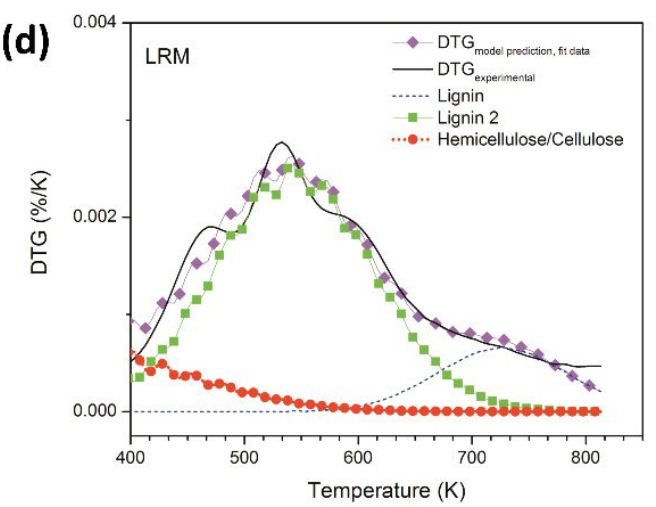

Figure S23. TG $(a, c)$ and DTG $(b, d)$ simulation results of three-parallel-DAEM model for CRM and LRM, respectively. The residuals between the observed and calculated mass data are also plotted for TG curves ( $a$ and $c$ ). 
Table S1. Yields of extract*

\begin{tabular}{|c|c|}
\hline Extraction media & Yield of extracts (\%) \\
\hline $\mathrm{MeOH} / \mathrm{H}_{2} \mathrm{O}(70: 30)$ & 8.2 \\
\hline $\mathrm{ChCl}: \mathrm{Oa} \times 2 \mathrm{H}_{2} \mathrm{O}(1: 1)$ & 33.7 \\
\hline ChCl:Ma (1:1) & 16.7 \\
\hline ChCl:Ca xH $\mathrm{H}_{2} \mathrm{O}(1: 1)$ & 10.5 \\
\hline ChCl:But (1:4) & 12.4 \\
\hline ChCl:EG (1:4) & 8.5 \\
\hline ChCl:Glu (1:1) & 18.7 \\
\hline ChCl:Glu (1:2) & 5.7 \\
\hline ChCl:Malt (1:1) & 10.7 \\
\hline
\end{tabular}

${ }^{*}$ Yield $\%=(\text { mass of dried phenolic extracts/mass initial mass of chestnut shells waste })^{*} 100$

Table S2. Interval range experimental data Identified by DDTG method to fit the three parallelDAEM model

\begin{tabular}{|c|c|c|c|c|}
\hline Sample & $\begin{array}{c}\text { Initial Normalized } \\
\text { mass from TG }\end{array}$ & $\mathbf{T}_{\mathbf{0}}$ & $\begin{array}{c}\text { Final Normalized } \\
\text { mass from TG }\end{array}$ & $\mathbf{T}_{\mathbf{f}}$ \\
\hline CSW & 0.9368 & 435 & 0.3132 & 848 \\
\hline CSWR & 0.9904 & 415 & 0.3994 & 820 \\
\hline CRM & 0.9884 & 415 & 0.3240 & 811 \\
\hline LRM & 0.9272 & 382 & 0.3673 & 809 \\
\hline
\end{tabular}


Table S3. Optimized kinetics parameters of first order three-DAEM-reaction model

\begin{tabular}{|c|c|c|c|c|c|c|c|}
\hline \multirow{2}{*}{ Sample } & \multirow{2}{*}{$\begin{array}{l}\text { Pseudo } \\
\text { component }\end{array}$} & \multicolumn{6}{|c|}{ First order reaction model } \\
\hline & & $\begin{array}{c}A_{i} \\
\left(\mathbf{s}^{-1}\right)\end{array}$ & $\begin{array}{c}E_{i} \\
(k J / m o l)\end{array}$ & $\left.\begin{array}{c}\sigma \\
(k J / m o l\end{array}\right)$ & c & Fit (\%) & $\begin{array}{l}\text { Final } \\
\text { O.F. } \\
\text { value }\end{array}$ \\
\hline \multirow{3}{*}{ csw } & 1 & $3.38 \times 10^{13}$ & 56.04 & 13.23 & 0.0881 & \multirow{3}{*}{$\begin{array}{c}0.366 \\
(n p=4957)^{*}\end{array}$} & \multirow{3}{*}{0.0585} \\
\hline & 2 & $4.34 \times 10^{15}$ & 196.51 & 15.79 & 0.5248 & & \\
\hline & 3 & $2.57 \times 10^{15}$ & 257.34 & 19.35 & 0.3871 & & \\
\hline \multirow{3}{*}{ CSWR } & 1 & $8.30 \times 10^{13}$ & 185.77 & 27.80 & 0.5207 & \multirow{3}{*}{$\begin{array}{c}0.499 \\
(\mathrm{np}=4836)^{*}\end{array}$} & \multirow{3}{*}{0.1180} \\
\hline & 2 & $8.63 \times 10^{14}$ & 197.10 & 4.06 & 0.4124 & & \\
\hline & 3 & $9.45 \times 10^{15}$ & 267.08 & 16.10 & 0.0669 & & \\
\hline \multirow{3}{*}{ CRM } & 1 & $8.63 \times 10^{15}$ & 190.69 & 21.27 & 0.2140 & \multirow{3}{*}{$\begin{array}{c}0.527 \\
(\mathrm{np}=4833)^{*}\end{array}$} & \multirow{3}{*}{0.1313} \\
\hline & 2 & $8.78 \times 10^{13}$ & 189.36 & 3.27 & 0.5659 & & \\
\hline & 3 & $1.11 \times 10^{16}$ & 258.74 & 20.43 & 0.2202 & & \\
\hline \multirow{3}{*}{ LRM } & $1^{*}$ & $3.57 \times 10^{14}$ & 118.47 & 29.92 & 0.1878 & \multirow{3}{*}{$\begin{array}{c}0.308 \\
(\mathrm{np}=5254)^{*}\end{array}$} & \multirow{3}{*}{0.043} \\
\hline & 2 & $4.36 \times 10^{12}$ & 154.40 & 19.67 & 0.6657 & & \\
\hline & 3 & $9.43 \times 10^{14}$ & 239.64 & 17.81 & 0.1465 & & \\
\hline
\end{tabular}

- $\mathrm{np}=$ =number of experimental points used for the fitting

- $1=$ Hemicellulose, $2=$ cellulose, $3=$ lignin, $1^{*}=$ lignin oligomers 